Open Access Full Text Article

ORIGINAL RESEARCH

\title{
Improved Tear Film Stability in Patients with Dry Eye After Hyaluronic Acid and Galactoxyloglucan Use
}

This article was published in the following Dove Press journal: Clinical Ophthalmology

\author{
Pedro Molina-Solana (ID) \\ Francisco de Borja \\ Domínguez-Serrano (D) \\ Antonio Manuel \\ Garrido-Hermosilla $\mathbb{D}^{1,2}$ \\ Jesús Montero-Iruzubieta $\mathbb{D}^{1,2}$ \\ Ana Fernández-Palacín (1D ${ }^{3}$ \\ Enrique Rodríguez-de-la-Rúa- \\ Franch $^{1,2}$ \\ Manuel Caro-Magdaleno (D) ${ }^{1,2}$ \\ 'Ophthalmology, Virgen Macarena \\ Hospital Seville, Seville, Spain; ${ }^{2}$ Surgery \\ Department, University of Seville, Seville, \\ Spain; ${ }^{3}$ Preventive Medicine and Public \\ Health Department, University of Seville, \\ Seville, Spain
}

Purpose: Artificial tears only provide transient relief for dry eye. To the best of our knowledge, this is the first study to objectively compare treatment with artificial tears with Keratograph 5M, which allows accurate and objective investigation of dry eye and artificial tear treatment. We aimed to evaluate whether a preservative-free combination of $0.4 \%$ hyaluronic acid and $0.2 \%$ galactoxyloglucan can improve dry eye using the new topographer, Keratograph 5M.

Patients and Methods: This prospective longitudinal, single-arm interventional cohort study was performed at a tertiary referral center and involved 20 patients with dry eye (40 eyes). Preservative-free artificial tears were administered every $3 \mathrm{~h}$. The participants underwent clinical and instrumental evaluations at baseline, 15, 30, 60, 90 and $120 \mathrm{~min}$ after instillation and 1 week and 1 month after treatment. Baseline values were considered as the controls. All patients were assessed with Keratograph 5M for non-invasive Keratograph first break-up time and non-invasive Keratograph average break-up time, conjunctival hyperemia, and tear meniscus height. Ocular surface staining with fluorescein was evaluated using the slit-lamp and fluorescein break-up time examinations, and the Ocular Surface Disease Index score was recorded for each patient.

Results: The signs and symptoms improved after 1 month of preservative-free $0.4 \%$ hyaluronic acid and $0.2 \%$ galactoxyloglucan treatment. There was a significant increase in the non-invasive Keratograph first break-up time and non-invasive Keratograph average break-up time at 15,30,60, and $90 \mathrm{~min}$, and 1 week and 1 month $(\mathrm{P}<0.05)$ and a decrease in hyperemia, corneal staining, and Ocular Surface Disease Index scores after 1 week and 1 month $(\mathrm{P}<0.05)$. No treatment-related adverse event was observed.

Conclusion: A combination of $0.4 \%$ hyaluronic acid and $0.2 \%$ galactoxyloglucan artificial tears seems effective for treating dry eye. Keratograph $5 \mathrm{M}$ can objectively detect these changes during the follow-up period.

Keywords: artificial tears, Keratograph, non-invasive Keratograph break-up time, Ocular Surface Disease Index, dry eye

\section{Introduction}

Dry eye is one of the most common pathologies encountered in ophthalmologic practice and with an estimated prevalence of $14-35 \%$ in adults. ${ }^{1,2}$ Artificial tears are used worldwide, to treat the signs and symptoms of dry eye, and the dosage depends on the intensity of the symptoms. However, these treatments only provide transient relief, as the artificial tears do not treat the underlying condition.
Correspondence: Manuel Caro-

Magdaleno

Calle Bogotá 7, Bloque 3, $1^{\circ} \mathrm{B}$, Seville

41013 , Spain

Tel +34609030403

Email mcaro79@gmail.com 
The preservative-free combination of $0.4 \%$ hyaluronic acid (average $8.5 \times 10^{5} \mathrm{Da}$ ) obtained from bacterial fermentation and $0.2 \%$ galactoxyloglucan $\left(6 \times 10^{5} \mathrm{Da}\right)$, which is buffered with sodium citrate and citric monohydrate acid, is a newly marketed lacrimal substitute. Both the active ingredients are highly mucoadhesive polymers with no Newtonian rheology and a high molecular weight. These properties of the ingredients act synergistically to protect the ocular surface. ${ }^{3,4}$ Hyaluronic acid allows for the retention of water that is up to 1000 -fold greater than its own weight and has a better precorneal residence time than low viscous solutions. ${ }^{5}$ Moreover, galactoxyloglucan is a natural polysaccharide derived from tamarind seeds with protective barrier properties. Moreover, it increases the corneal wound healing rate and reduces the in vitro toxicity of timolol, thiomersal, and fluoroquinolones in human conjunctival cells. ${ }^{6}$ It can improve some ocular surface abnormalities in patients with glaucoma, caused by exposure to prostaglandins and benzalkonium chloride, thereby, protecting the ocular surface. ${ }^{7}$

The purpose of our study was to investigate the effect of these artificial tears on dry eye by measuring the following parameters using the topographer, Keratograph 5M (OculusOptikgeräte GmbH, Wetzlar, Germany): Primary, non-invasive Keratograph first tear break-up time (time elapsed in seconds from the last blink until the first break in the tear film) and non-invasive Keratograph average break-up time (average time of all the tear film breakups) during the first $120 \mathrm{~min}$ after instillation and after 1 month of treatment. The secondary objective, was to investigate the effect of these artificial tears taking measurements of conjunctival hyperemia, tear meniscus height and also the Ocular Surface Disease Index and Oxford scores using slit-lamp examination. The reliability and repeatability of the measurements obtained by Keratograph $5 \mathrm{M}$ have been validated by earlier investigations, performing objective measures. ${ }^{8-10}$

\section{Patients and Methods}

https://dx.doi.org/10.17504/protocols.io.bbxtipnn

\section{Participant Recruitment}

The sample size was determined by a statistically significant difference of $95 \%$ confidence intervals and with $80 \%$ power for non-invasive Keratograph break-up time based on a pilot study. A total of 34 eyes was required, according to the program nQuery Advisor Release 7.0 (Statistical Solutions, Broadway, Saugus, Massachusetts, USA). The study included 40 eyes from 20 participants (18 women and 2 men; age range, 36 to 79 years) with signs and symptoms of dry eye, who visited our hospital between November and December 2017. The results of the Schirmer I test without anesthesia and fluorescein breakup time test were recorded at the time of the visit, and the patient was enrolled in the study if the result was $<10 \mathrm{~mm}$ at $5 \mathrm{~min}$ or $<10 \mathrm{~s}$, respectively. Moreover, only participants who were older than 18 years, did not have any active ocular surface disease, who did not wear contact lenses for at least $24 \mathrm{~h}$ prior to the visit, did not undergo any ocular surgery at least 1 year before the visit, who were not pregnant or lactating, and did not have any known allergy to any of the components of the tear supplement were included. Patients who failed to meet these criteria were excluded from the study. Written informed consent was obtained from all participants. This research was approved by the Ethical Committee of Virgen Macarena's Hospital and was conducted according to the tenets of the Declaration of Helsinki. The data recorded was confidential.

All enrolled patients visited the hospital thrice. They visited the hospital at approximately the same hour during the day and in the same examination room. The temperature and humidity of the examination room were maintained at around $22-25^{\circ} \mathrm{C}$ and $45-55 \%$ humidity, respectively. All patients completed the study, and there were no withdrawals. During the follow-up month, all patients were treated with artificial tears composed of $0.4 \%$ hyaluronic acid and $0.2 \%$ galactoxyloglucan every $3 \mathrm{~h}$ and were allowed to rest at night. The patients were questioned about compliance at every visit. The patients were asked to continue with any other medication (topical steroids or topical immunosuppressants), to prevent masking the true effect of the artificial tears. Only patients who were using other artificial tears were instructed to switch to the artificial tears used in our study maintaining previous treatments.

\section{Ocular Examinations}

Details regarding previous or current topical or systemic treatment were recorded during the first visit, and the following parameters were measured in each eye using the topographer, Keratograph 5M (OculusOptikgeräte $\mathrm{GmbH}$, Wetzlar, Germany): tear meniscus height (3 measurements were made manually along the length of the lower eyelid using the cursors provided by the system, and the average of the 3 values was considered), conjunctival 
hyperemia (indicated by the software of the Keratograph $5 \mathrm{M}$; range, 0.1 to 4.0 ; accuracy, 0.1 ), non-invasive Keratograph first break-up time (moment at which the tear film breaks for the first time) and non-invasive Keratograph average break-up time (average time of the first rupture at different locations of the 8-mm diameter of the central cornea), and the Ocular Surface Disease Index questionnaire (Allergan Inc, Irvine, CA, USA). Corneal staining results (using the Oxford schema) ${ }^{11}$ and fluorescein break-up time were evaluated using slit-lamp examination. Baseline measurements were used as controls: each patient was his/her own control. After a 30-min washout period, a drop of the artificial tears was instilled into the inferior palpebral fold of both eyes. The noninvasive Keratograph first break-up time and noninvasive Keratograph average break-up time values were measured at $15 \mathrm{~min}, 30 \mathrm{~min}, 60 \mathrm{~min}, 90 \mathrm{~min}$, and $120 \mathrm{~min}$ after artificial tear instillation or until the non-invasive Keratograph average break-up time value was less than the baseline value. The objective of this assessment was to verify the time required for the artificial tear to bring about an increase in the non-invasive Keratograph break-up time parameters in the patients.

During the second visit, which took place 1 week after the first, and the third visit, which took place 3 weeks after the second, the tear meniscus height, conjunctival hyperemia, non-invasive Keratograph first break-up time and non-invasive Keratograph average break-up time, and Ocular Surface Disease Index score were measured again. Corneal staining and fluorescein break-up time were assessed using slit-lamp examination.

\section{Statistical Analysis}

Data were analyzed using IBM SPSS Statistics 24.0@) Statistical analyses included descriptive analysis, with means, medians, standard deviations, and 25th and 75th percentiles (in case of marked asymmetry) for quantitative variables and percentages for qualitative variables. Inferential analysis consisted of a repeated-measures analysis of variance (ANOVA) to test differences between the variables of conjunctival hyperemia, Oxford and Ocular Surface Disease Index scores, tear meniscus height, lacrimal stability level, non-invasive Keratograph first breakup time and non-invasive Keratograph average break-up time recorded during each follow-up visit. The Bonferroni test was used to detect parameters exhibiting significant differences. The least significant difference test was used in case of inconsistency between the ANOVA and
Bonferroni values. $P$-values $<0.05$ were considered to be indicative of statistical significance, and the results were presented as mean \pm standard deviation.

\section{Results}

Twenty patients were enrolled for the study. All the enrolled patients followed our treatment schedule. The adverse effects of the treatment included blurred vision a few minutes after instillation [6 patients (30\%)] and ocular stinging [3 patients (15\%)]. None of the patients experienced adverse reactions that required discontinuation of treatment.

Thirty-two eyes $(80 \%)$ had a Schirmer I test result of $<10 \mathrm{~mm}$, and the remaining 8 eyes $(20 \%)$ had a tear break-up time of $<10 \mathrm{~s}$. Seven patients (35\%) had not undergone any previous treatment at the beginning of the study, $10(50 \%)$ were being treated with different artificial tears, $2(10 \%)$ were using artificial tears and topic steroids, and 1 (5\%) was being treated with topical immunosuppressants in addition to artificial tears and topical steroids.

The baseline, 1-week, and 1-month demographic and clinical data of the patients are summarized in Table 1. Noninvasive Keratograph first break-up time and non-invasive Keratograph average break-up time were significantly higher at 1 week $(P=0.01$ and $P=0.007$, respectively) and 1 month after commencing treatment $(P=0.003$ and $P=0.002$, respectively) than those at baseline (Figure 1). Tear meniscus height was significantly higher at 1 week $(P=0.002)$ and at 1 month $(P=0.001)$ than that at baseline. Conjunctival hyperemia reduced significantly at 1 month $(P=0.008)$ (Figure 2), and the Oxford and Ocular Surface Disease Index scores were significantly lower at 1 week $(P=0.03$ and $P<0.0001$, respectively) and 1 month $(P<0.0001$ and $P<0.0001$, respectively), compared to those at baseline (Figure 3).

The analysis of the results showed a statistically significant increase in non-invasive Keratograph first breakup time during the first $60 \mathrm{~min}$ after instillation of the artificial tears $(P=0.004$ at $15 \mathrm{~min}, P=0.03$ at $30 \mathrm{~min}$, and $P=0.01$ at $60 \mathrm{~min}$ ).

Non-invasive Keratograph average break-up time was significantly increased till $90 \mathrm{~min}$ after instillation $(P=0.01$ at $15 \mathrm{~min}, P<0.0001$ at $30 \mathrm{~min}, P=0.002$ at $60 \mathrm{~min}$, and $P=0.03$ at $90 \mathrm{~min}$ ). (Figure 4 )

\section{Discussion}

The present study demonstrated the effectiveness of preservative-free artificial tears containing $0.4 \%$ hyaluronic acid and $0.2 \%$ galactoxyloglucan in patients with signs 
Table I Baseline, I-Week, and I-Month Clinical and Demographic Characteristics of the Participants

\begin{tabular}{|c|c|c|c|}
\hline & Baseline & I Week & I Month \\
\hline Age (years) & $63.85 \pm 13.15$ & & \\
\hline $\operatorname{Sex}(M: F)$ & $2: 18$ & & \\
\hline $\begin{array}{l}\text { Schirmer I test } \\
(\mathrm{mm} / 5 \mathrm{~min})\end{array}$ & $7.53 \pm 4.69$ & & \\
\hline FBUT (s) & $4.80 \pm 2.14$ & $\begin{array}{l}7.20 \pm 2.51 \\
P=0.000\end{array}$ & $\begin{array}{l}8.73 \pm 2.26 \\
P=0.000\end{array}$ \\
\hline TMH (mm) & $0.27 \pm 0.10$ & $\begin{array}{l}0.33 \pm 0.13 \\
P=0.002\end{array}$ & $\begin{array}{l}0.33 \pm 0.15 \\
P=0.001\end{array}$ \\
\hline NIKBUT-first (s) & $6.20 \pm 4.59$ & $\begin{array}{l}8.35 \pm 5.26 \\
P=0.014\end{array}$ & $\begin{array}{l}9.52 \pm 5.92 \\
P=0.003\end{array}$ \\
\hline NIKBUT-average (s) & $10.02 \pm 5.72$ & $\begin{array}{l}12.12 \pm 5.52 \\
P=0.007\end{array}$ & $\begin{array}{l}13.22 \pm 5.30 \\
P=0.002\end{array}$ \\
\hline Conjunctival hyperemia & $1.36 \pm 0.54$ & $\begin{array}{l}1.19 \pm 0.45 \\
P=0.08\end{array}$ & $\begin{array}{l}1.12 \pm 0.48 \\
P=0.008\end{array}$ \\
\hline Oxford score & $2.68 \pm 2.53$ & $\begin{array}{l}2.03 \pm 1.76 \\
P=0.025\end{array}$ & $\begin{array}{l}0.95 \pm 1.78 \\
P=0.000\end{array}$ \\
\hline OSDI score & $37.58 \pm 17.66$ & $\begin{array}{l}29.69 \pm 18.67 \\
P=0.000\end{array}$ & $\begin{array}{l}16.41 \pm 8.56 \\
P=0.000\end{array}$ \\
\hline
\end{tabular}

Note: $P$-values represent the differences between the baseline and the subsequent timepoints.

Abbreviations: $M$, male; F, female; FBUT, fluorescein break-up time; $\mathrm{TMH}$, tear meniscus height; NIKBUT, non-invasive Keratograph tear break-up time; OSDI, Ocular Surface Disease Index.

and symptoms of dry eye using the Keratograph 5M, as there were improvements in all of the dry-eye-related parameters studied.

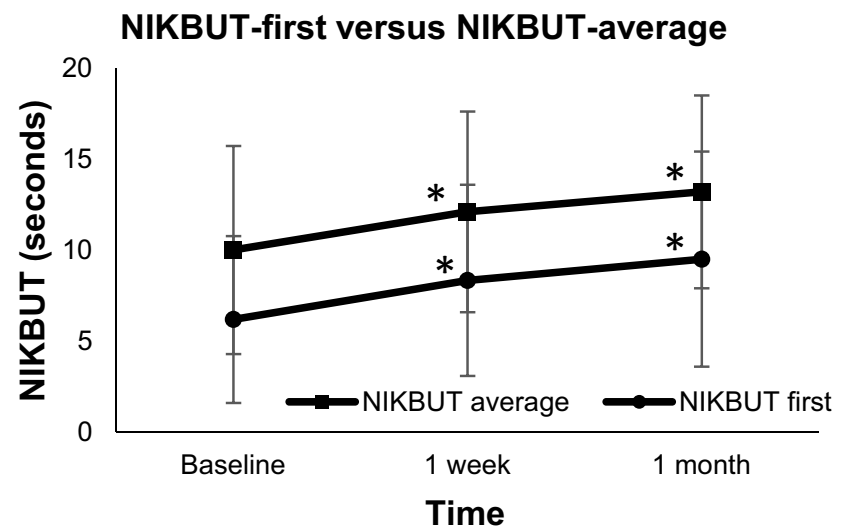

Figure I Comparison of the mean values of non-invasive Keratograph 5M first tear break-up time and non-invasive Keratograph average break-up time at the 3 visits, showing an increase at I week and I month from baseline. The asterisk (*) denotes statistical significance.

Abbreviation: NIKBUT, non-invasive Keratograph break-up time.
CONJUNCTIVAL HYPEREMIA

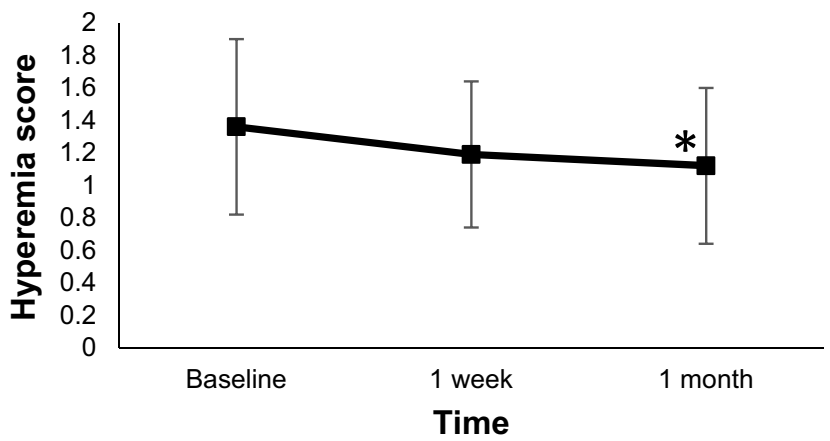

Figure 2 Mean hyperemia scores of all patients during the 3 visits. The asterisk (*) denotes statistical significance.

While earlier studies have measured and compared tear meniscus height using the Schirmer test and anterior segment swept-source optical coherence tomography, ${ }^{12,13}$ the measurement of this parameter with the Keratograph

\section{OXFORD}
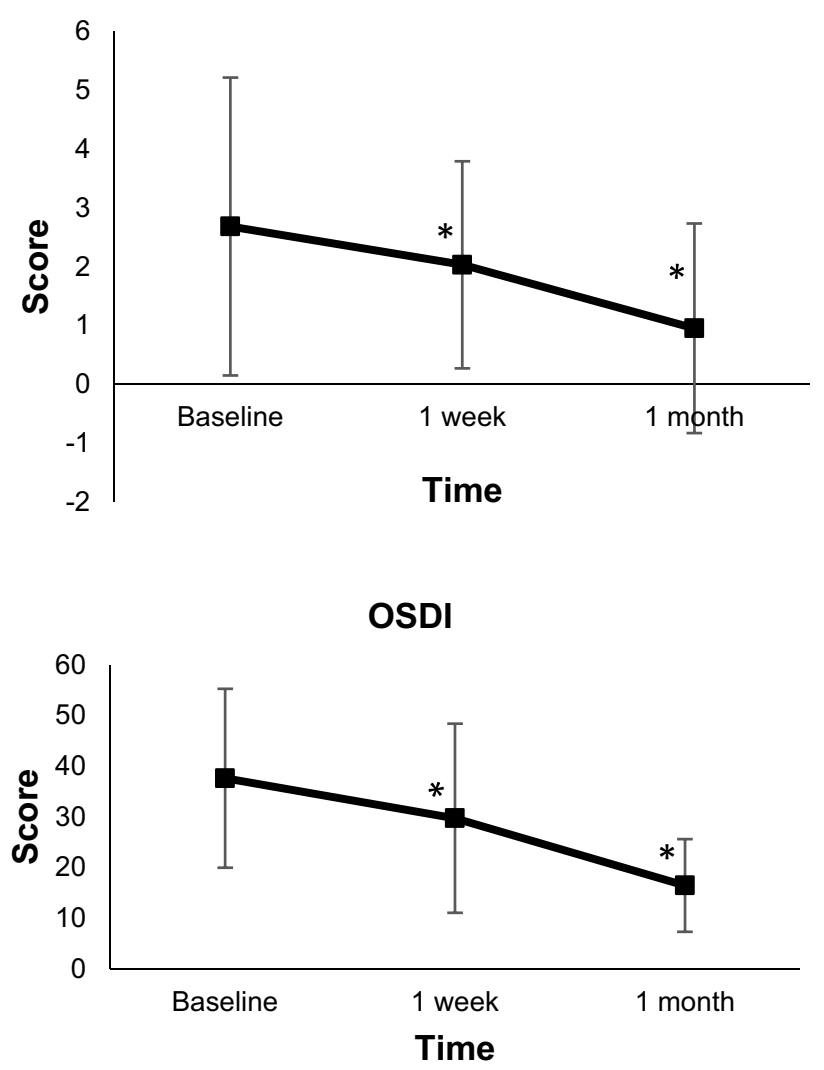

Figure 3 Mean values of the Oxford score measured during slit-lamp examination (top panel) and the Ocular Surface Disease Index scores (bottom panel) recorded during the study, showing a decrease at I week and I month from baseline. The asterisk $(*)$ denotes statistical significance.

Abbreviation: OSDI, Ocular Surface Disease Index. 


\section{NIKBUT-first versus NIKBUT-average}

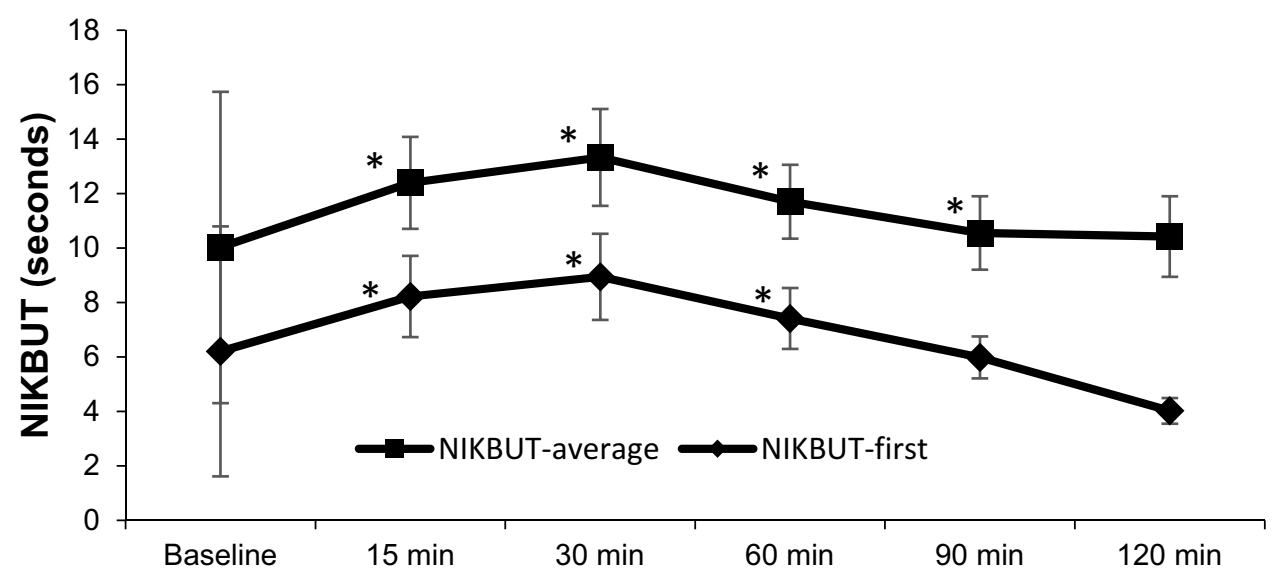

Time (minutes)

Figure 4 Comparison of the mean values of non-invasive Keratograph $5 \mathrm{M}$ first tear break-up time and non-invasive Keratograph average break-up time measured during the first I 20 min after instillation of the drop. There was an initial increase in both parameters at I5 min, followed by a gradual decrease after 30 min. The asterisk (*) denotes statistical significance.

Abbreviation: NIKBUT, Non-invasive Keratograph break-up time.

exhibits high reliability and repeatability. ${ }^{14}$ In our study, we observed a significant increase in tear meniscus height at 1 week and 1 month.

Traditionally, conjunctival hyperemia is assessed using visual scales, ${ }^{15}$ which are subjective, while the analysis of conjunctival hyperemia using the Keratograph, is a quick and easy process. Moreover, previous studies have measured conjunctival hyperemia using Keratograph in patients who were treated with antiglaucoma eye drops. ${ }^{16}$ Our results showed a statistically significant decrease in the bulbar redness scores at 1 month, while no significant reduction was observed at 1 week, suggesting that prolonged treatment is required to achieve a significant decrease in bulbar redness. This is similar to the finding of an earlier study on antiglaucoma drops, which reported a decrease in the bulbar redness scores at 6 months in patients who used preservative-free antiglaucoma drops. ${ }^{17}$

The present study involved the measurement of 2 types of non-invasive Keratograph break-up time: non-invasive Keratograph first break-up time and non-invasive Keratograph average break-up time. The fact that the noninvasive Keratograph first break-up time and non-invasive Keratograph average break-up time values were recorded during the first 120 min after the instillation of 1 drop of the preservative-free artificial tear represents the significance and novelty of our study. The results showed that non-invasive Keratograph first break-up time was higher when compared to the baseline value till $60 \mathrm{~min}$ after instillation, and that non-invasive Keratograph average break-up time was higher, when compared to the baseline value till $90 \mathrm{~min}$ after instillation.

The improvement in non-invasive Keratograph breakup time values represents a crucial aspect in the treatment of dry-eye syndrome. ${ }^{18}$ This improvement correlates well with the corneal staining finding, as well as the Oxford and Ocular Surface Disease Index scores obtained in the present study, where a reduction in the above-mentioned parameters was observed at 1 week. Previous studies on patients with evaporative dry eye reported that the noninvasive Keratograph break-up time was higher at 1 and 3 months, and 4 weeks of treatment compared to that at baseline. ${ }^{19,20}$ These results are concurrent with our findings at 1 month after treatment.

The objective measurements obtained with the Keratograph 5M using infrared light were the strength of our study, thus, eliminating the need for fluorescein staining or topical anesthesia, which alter the lacrimal film. ${ }^{21}$

To the best of our knowledge, this is one of the first studies to compare artificial tear treatment objectively with Keratograph $5 \mathrm{M},{ }^{22}$ which enabled accurate and objective ophthalmologic investigations not being necessary photographs and scales nor subjective methods like TBUT. However, further investigations are needed to validate these findings.

Furthermore, the absence of a placebo group is a limitation of the study and its inclusion should be 
considered in future studies. Moreover, future studies could include larger sample populations and study one eye per patient, to confirm the results. Moreover, a compliance form filled not only by the investigator during follow-up visits, but also by patients could reduce the variability in the implementation of the artificial tear regimen and should be considered in future studies.

In conclusion, artificial tears composed of preservativefree $0.4 \%$ hyaluronic acid and $0.2 \%$ galactoxyloglucan are useful for the treatment of the signs and symptoms of dry eye, improving NIKBUT, conjunctival hyperemia, Oxford and OSDI scores after 1 month of treatment.

\section{Acknowledgment}

The artificial tears used in the study were donated by Esteve Pharmaceuticals.

\section{Disclosure}

IATA and RETICS OFTARED have sponsored the translation of this manuscript. AMG-H, JM-I, ER-d-1-R-F, and MC-M are members of RETICS OFTARED 'RD16/0008/ $0010^{\prime}$, financed by Instituto de Salud Carlos III, as part of the Plan Nacional I+D+i 2013-2016 and co-financed by the European Union (FEDER/FSE) "Una manera de hacer Europa." None of the funding agencies played an active role in the study design and in the decision to submit this manuscript for publication, and they do not have ultimate authority over any of these activities. The authors report no other possible conflicts of interest in this work.

\section{References}

1. Moss SE, Klein R, Klein BE. Prevalence of and risk factors for dry eye syndrome. Arch Ophthalmol. 2000;118(9):1264-1268. doi:10.1001/ archopht.118.9.1264

2. Paulsen AJ, Cruickshanks KJ, Fischer ME, et al. Dry eye in the beaver dam offspring study: prevalence, risk factors, and health-related quality of life. Am J Ophthalmol. 2014;157(4):799-806. doi:10.1016/j. ajo.2013.12.023

3. Uccello-Barretta G, Nazzi S, Zambito Y, Di Colo G, Balzano F, Sansò M. Synergistic interaction between TS-polysaccharide and hyaluronic acid: implications in the formulation of eye drops. Int J Pharm. 2010;395(1-2):122-131. doi:10.1016/j.ijpharm.2010.05.031

4. Versura P, Profazio V, Balducci N, Campos EC. Efficacy of two-month treatment with Xiloial eyedrops for discomfort from disposable soft contact lenses. Clin Ophthalmol. 2010;4:1035-1041. doi:10.2147/ OPTH.S10448

5. Mateo Orobia AJ, Saa J, Ollero Lorenzo A, Herreras JM. Combination of hyaluronic acid, carmellose, and osmoprotectants for the treatment of dry eye disease. Clin Ophthalmol. 2018;12:453-461. doi:10.2147/ OPTH.S157853

6. Soumendra S, Rashmirekha S, Padma LN. Tamarind seed polysaccharide: a versatile biopolymer for mucoadhesive applications. J Pharm Biomed Sci. 2010;8:20.
7. Iester M, Oddone F, Fogagnolo P, Frezzotti P, Figus M. Confocal Microscopy Study Group. Changes in the morphological and functional patterns of the ocular surface in patients treated with prostaglandin analogues after the use of TSP $0.5 \%{ }^{\circledR}$ preservative-free eyedrops: a prospective, multicenter study. Ophthalmic Res. 2014;51(3):146-152. doi:10.1159/000357100

8. Cox SM, Nichols KK, Nichols JJ. Agreement between automated and traditional measures of tear film breakup. Optom Vis Sci. 2015;92(9): e257-63. doi:10.1097/OPX.0000000000000648

9. Wu S, Hong J, Tian L, Cui X, Sun X, Xu J. Assessment of bulbar redness with a newly developed keratograph. Optom Vis Sci. 2015;92 (8):892-899. doi:10.1097/OPX.0000000000000643

10. Markoulli M, Duong TB, Lin M, Papas E. Imaging the tear film: a comparison between the subjective keeler tearscope-plus ${ }^{\mathrm{TM}}$ and the objective oculus ${ }^{\circledR}$ keratograph $5 \mathrm{M}$ and LipiView ${ }^{\circledR}$ interferometer. Curr Eye Res. 2018;43(2):155-162. doi:10.1080/0271 3683.2017.1393092

11. Bron A, Evans VE, Smith JA. Grading of corneal and conjunctival staining in the context of other dry eye tests. Cornea. 2003;22 (7):640-650. doi:10.1097/00003226-200310000-00008

12. Akiyama-Fukuda R, Usui T, Yoshida T, Yamagami S. Evaluation of tear meniscus dynamics using anterior segment swept-source optical coherence tomography after topical solution instillation for dry eye. Cornea. 2016;35(5):654-658. doi:10.1097/ICO.00000000 00000807

13. Baek J, Doh SH, Chung SK. Comparison of tear meniscus height measurements obtained with the keratograph and fourier domain optical coherence tomography in dry eye. Cornea. 2015;34 (10):1209-1213. doi:10.1097/ICO.0000000000000575

14. López-de la Rosa A, Pinto-Fraga J, Blázquez Arauzo F, Urbano Rodríguez R, González-García MJ. Safety and efficacy of an artificial tear containing $0.3 \%$ hyaluronic acid in the management of moderate-to-severe dry eye disease. Eye Contact Lens. 2017;43 (6):383-388. doi:10.1097/ICL.0000000000000284

15. Nagahara Y, Koh S, Nishida K, et al. Prolonged increase in tear meniscus height by $3 \%$ diquafosol ophthalmic solution in eyes with contact lenses. Clin Ophthalmol. 2015;9:1029-1031. doi:10.2147/ OPTH.S86173

16. Pérez-Bartolomé F, Martínez-de-la-Casa JM, Arriola-Villalobos P, et al. Ocular redness measured with the keratograph $5 \mathrm{~m}$ in patients using anti-glaucoma eye drops. Semin Ophthalmol. 2018;33 (5):643-650. doi:10.1080/08820538.2017.1395891

17. El Ameen A, Vandermeer G, Pisella PJ. Efficacy and patient tolerability of preservative-free latanoprost compared with preservative prostaglandin analogs in patients with ocular hypertension or glaucoma. Acta Ophthalmol. 2016;94.

18. Abdelfattah NS, Dastiridou A, Sadda SR, Lee OL. Noninvasive imaging of tear film dynamics in eyes with ocular surface disease. Cornea. 2015;34:S48-52. doi:10.1097/ICO.0000000000000570

19. Kim YH, Kang YS, Lee HS, Choi W, You IC, Yoon KC. Effectiveness of combined tear film therapy in patients with evaporative dry eye with short tear film breakup time. J Ocul Pharmacol Ther. 2017;33(8):635-643. doi:10.1089/jop.2017.0019

20. Mun Y, Kwon JW, Oh JY. Therapeutic effects of 3\% diquafosol ophthalmic solution in patients with short tear film break-up time-type dry eye disease. BMC Ophthalmol. 2018;18(1):237. doi:10.1186/s12886-018-0910-3

21. Koh S, Ikeda C, Watanabe S, et al. Effect of non-invasive tear stability assessment on tear meniscus height. Acta Ophthalmol. 2015;93(2):e135-139. doi:10.1111/aos.12516

22. Markoulli M, Sobbizadeh A, Tan J, Briggs N, Coroneo M. The effect of optive and optive advanced artificial tears on the healthy tear film. Curr Eye Res. 2018;43(5):588-594. doi:10.1080/02713683.2018. 1433860 


\section{Publish your work in this journal}

Clinical Ophthalmology is an international, peer-reviewed journal covering all subspecialties within ophthalmology. Key topics include: Optometry; Visual science; Pharmacology and drug therapy in eye diseases; Basic Sciences; Primary and Secondary eye care; Patient Safety and Quality of Care Improvements. This journal is indexed on PubMed

Submit your manuscript here: https://www.dovepress.com/clinical-ophthalmology-journal
Central and CAS, and is the official journal of The Society of Clinical Ophthalmology (SCO). The manuscript management system is completely online and includes a very quick and fair peer-review system, which is all easy to use. Visit http://www.dovepress.com/ testimonials.php to read real quotes from published authors. 